\title{
Evaluation of Child Development Centre Based Intervention for Children with Multiple Disability
}

\author{
Mustafa Mahbub ${ }^{1}$, Humaira Rafiqa², Dilara Begum ${ }^{3}$ \\ ${ }^{1}$ Professor \& Head, Department of Pediatric Neuroscience, Bangladesh Institute of Child Health \& Dhaka Shishu Hospital, \\ Dhaka, Bangladesh; ${ }^{2}$ Assistant Professor, Pediatric Neuroscience Department, Bangladesh Institute of Child Health \& \\ Dhaka Shishu Hospital, Dhaka, Bangladesh; ${ }^{3}$ Developmental Therapist, Department of Pediatric Neuroscience, \\ Bangladesh Institute of Child Health \& Dhaka Shishu Hospital, Dhaka, Bangladesh
}

[Received on: 22 November 2020; Accepted on: 12 December 2020; Published: 1 January 2021]

\begin{abstract}
Background: Children with multiple disabilities usually have more than one significant disability that may include speech, physical mobility, learning, mental retardation, visual, hearing, and possibly others. Objective: The purpose of the present study was to evaluate the types of multiple disabilities in children with cerebral palsy and epilepsy and their fate after the intervention. Methodology: This retrospective observational study was conducted at Dhaka Shishu hospital from January 2011 to Decemberb2011.Children with multiple disabilities were referred to the child development center of Dhaka Shishu Hospital and were assessed for impairments and appropriate intervention done in special clinics. Result: Out of 540 children $66 \%$ were male. $43 \%$ of children were age group from 12-23 months. 58\% had tetraplegic cerebral palsy (CP) with epilepsy and the second-highest group was diplegic CP with epilepsy 75(14.0\%). Among 72 selected children who came in regular follow up, all children had motor disabilities. Cognitive delay, speech delay, vision and hearing impairment were found in 50\%, 79\%, 58\% and 54\% respectively. After intervention more improvement was found in motor function (92\%). Conclusion: Intervention in specialized clinics improved the quality of life of children with multiple disability. [Journal of National Institute of Neurosciences Bangladesh, January 2021;7(1): 38-41]
\end{abstract}

Keywords: : Multiple disabilities; cerebral palsy; intervention

Correspondence: Dr. Mustafa Mahbub, Professor \& Head, Department of Paediatric Neuroscience, Dhaka Shishu (Children's) Hospital, Sher-E-Bangla Nagar, Dhaka-1207, Bangladesh; Cell no.: +8801713209069; Email: mm231956@gmail.com

Conflict of interest: There is no financial conflict of interest relevant to this paper to disclose.

Funding agency: This research project was not funded by any group or any institution.

Contribution to authors: Mahbub M, Rafiqa $\mathrm{H}$ contributed from the protocol preparation, data collection up to report writing. Manuscript writing was performed by Mahbub M, Begum D; Rafiqa $\mathrm{H}$ has revised the manuscript.

How to cite this article: Mahbub M, Rafiqa H, Begum D. Evaluation of Child Development Centre Based Intervention for Children with Multiple Disability. J Natl Inst Neurosci Bangladesh, 2021;7(1): 38-41

Copyright: (C2021. Mahbub et al. Published by Journal of National Institute of Neurosciences Bangladesh. This article is published under the Creative Commons CC BY-NC License (https://creativecommons.org/licenses/by-nc/4.0/). This license permits use, distribution, and reproduction in any medium, provided the original work is properly cited and is not used for commercial purposes.

\section{Introduction}

Children with multiple disabilities will have a combination of various disabilities that may include issues with: speech, physical mobility, learning, mental retardation, sight, hearing, brain injury, and possibly others. Along with multiple disabilities, they can also exhibit sensory losses as well as behavior and/or social problems $^{1}$. Child with multiple disabilities have unique needs and challenges. Many of these young children struggle to communicate their wants and needs, to freely move their body to access and engage their world, and to learn abstract concepts and ideas ${ }^{2}$. Four areas of need medical, physical, learning, and social-emotional needs should be addressed in developing interventions for young children with multiple disabilities ${ }^{2}$.

Early intervention is necessary as soon as possible with the involvement of the appropriate professionals, i.e. occupational therapists, speech or language therapists, physiotherapists' like'. In children with cerebral palsy (CP), lesions of the central nervous system (CNS) could 
cause motor-sensory impairments that progressively deteriorate over time. The primary challenge for $\mathrm{CP}$ is gross motor dysfunction ${ }^{3,4}$. The motor problems of $\mathrm{CP}$ arise fundamentally from CNS dysfunction, which interferes in the development of normal postural control against gravity and impedes normal motor development $^{5-7}$. Occupational therapy in children with $\mathrm{CP}$ is performed to avoid abnormal muscle tone and posture, to treat muscle and joint deformities, and to reduce motor and sensory disorders ${ }^{8-9}$. In multiple disabilities, we take into consideration that the evaluation and intervention must be carried out individually as each person is considered unique according to the developmental profile $^{10}$. The intervention begins with early communication with children with multiple disabilities includes mutual attention, shared topic, common language, comfortable pace, turn-taking, and balance of turns, comfortable positioning, and mutual caring ${ }^{11}$. The development of communication involves interaction. The caregiver must be aware of the child's signals and behaviors and forms of communication, interpret them accurately, and give a response so that The Child feels that he is understood. Studies of intensive interaction have shown that this approach determined an increase in the children's attention to their interaction and communication partner, an increase of positive affect, proximity, and attention to the wider environment ${ }^{12}$. Many children with multiple disabilities exhibit tone problems. Specifically, their tone may be too high like hypertonia, which results in stiffness that interferes with movement, or muscle tone that is too low (i.e., hypotonic), which makes it difficult to stabilize joints in preparation for movement or to maintain a position. Children's muscle tone may also fluctuate from being too high to too low yet seldom in a state of normal tone. Abnormal muscle tone not only affects a child's ability to maintain and move within positions but also interferes with active movement and the use of one's extremities. Thus, professionals working with children with multiple disabilities may encounter numerous approaches to provide intervention directed toward these problems ${ }^{13}$.

Child Development Centre, Dhaka Shishu Hospital provides services for children with multiple disabilities by arranging special clinics such as multiple disability clinic (MDC), speech-language and communication clinic (SLC), sitting and feeding clinic, low vision clinic. Here a multi-disciplinary team consisting of a child health physician, psychologist, developmental therapist, and the parents and educational specialists, work together to plan and coordinate necessary services to be given to a child with multiple disabilities.

\section{Methodology}

This retrospective observational study was conducted at the Pediatric Neuroscience Department of Dhaka Shishu Hospital (DSH) from January 2011 to December 2011 for a period of one year. Children with multiple disabilities referred to the child development Centre of Dhaka Shishu Hospital were assessed for impairments and appropriate intervention was done in different clinics. In the child development center, there is a weekly multiple disability clinic. In addition to this clinic, CDC provides other specialized clinics such as more than words clinic, speech, language \& communication clinic, feeding and seating clinic, low vision clinic, and epilepsy clinic. The core team includes child health physicians, developmental therapists, and psychologists. For study purposes, children were classified as having multiple disabilities if they had two or more disabilities concurrently? ${ }^{7}$. We had searched from the record book and purposively selected the cases who had disabilities and came for follow up regularly. To assess the development of the child Rapid neurodevelopmental assessment (RNDA) tool was appilied ${ }^{14}$. Rapid neurodevelopmental assessment (RNDA) is an assessment tool designed to ascertain functional status like neurodevelopmental impairments (NDIs), across multiple neuro developmental domains; such as motor, cognition, vision, hearing, speech with grades of severity for each domain determined, so that appropriate intervention can be planned, and prioritized, according to the child's ascertained NDI profile. Data were analyzed in SPSS version 21.

\section{Results}

Out of 540 children, $66 \%$ were male and $43 \%$ of children were from the age group 12 to 23 months.

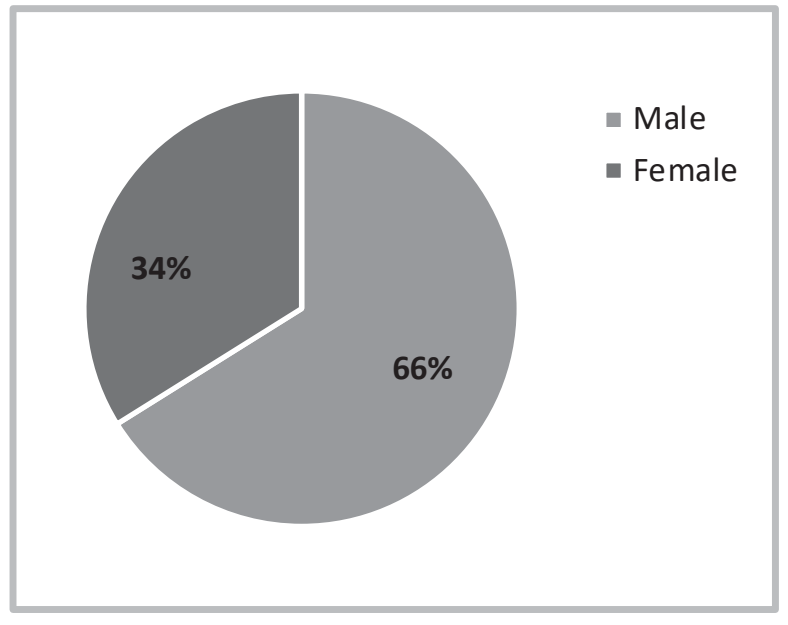

Figure I: Gender Distribution of the Study Patient 
Table 1: Age Group of Study Patient $(n=540)$

\begin{tabular}{lcc}
\hline Variables & Frequency & Percent \\
\hline Less Than 12 months & 87 & 16.0 \\
12 to 23 months & 234 & 43.0 \\
24 to 35 months & 138 & 26.0 \\
36 to 47 months & 36 & 7.0 \\
More Than 48 months & 45 & 8.0 \\
Total & $\mathbf{5 4 0}$ & $\mathbf{1 0 0 . 0}$ \\
\hline
\end{tabular}

Tetraplegic CP with epilepsy were more (58\%). Then other types of CP, like Diplegic CP, Hemiplegic CP, Dyskinetic CP were $14 \%, 11 \%$, and $11 \%$ respectively. (Table 2)

Table 2: Diagnosis of the Study Patients $(n=540)$

\begin{tabular}{lcc}
\hline Diagnosis & Frequency & Percent \\
\hline Tetraplegic CP with Epilepsy & 315 & 58 \\
Diplegic CP with Epilepsy & 75 & 14 \\
Hemiplegic CP with Epilepsy & 60 & 11 \\
Dyskinetic CP with Epilepsy & 60 & 11 \\
Seq. of Meningitis/Encephalitis & 12 & 2.5 \\
Chromosomal Syndrome & 9 & 2.0 \\
Neuro-metabolic Disorder & 3 & 0.5 \\
Erb's Palsy & 3 & 0.5 \\
Worster-Drought syndrome & 3 & 0.5 \\
\hline
\end{tabular}

Among 72 selected children who came in regular follow-up, the initial assessment showed that motor disability was present in all $72(100 \%)$ children. Cognitive delay, speech delay, vision and hearing impairment were found in $50 \%, 79 \%, 58 \%$ and $54 \%$ respectively. After intervention more improvement was found in motor function (92\%) (Table 3).

Table 3: Initial Impairment in Followed-Up Children $(\mathrm{n}=72)$

\begin{tabular}{lcc}
\hline Impairment & Frequency & Percent \\
\hline Motor & 72 & 100 \\
Speech & 57 & 79 \\
Feeding difficulties & 48 & 67 \\
Vision & 42 & 58 \\
Hearing & 39 & 54 \\
Cognition & 36 & 50 \\
Seizure & 33 & 45
\end{tabular}

*Percentages and totals based on multiple response analysis

Out of 72 children 66(92.0\%) cases improved in motor function, $27(38.0 \%)$ cases improved in cognitive function, $36(50.0 \%)$ cases improved in speech,
$30(43.0 \%)$ cases improved in feeding, 24(33.0\%) cases improved in vision and 24(33.0\%) cases improved in hearing (Table 4).

Table 4: Impairment VS Improvement $(n=72)$

\begin{tabular}{lccc}
\hline Disabilities & Impairment & Improvement & $\begin{array}{c}\text { Percent of } \\
\text { improvement }\end{array}$ \\
\hline Motor & 72 & 66 & $92 \%$ \\
Speech & 57 & 36 & $63 \%$ \\
Feeding & 48 & 30 & $63 \%$ \\
Vision & 42 & 24 & $57 \%$ \\
Hearing & 39 & 24 & $61 \%$ \\
Cognition & 12 & 27 & $75 \%$ \\
Seizure & 33 & Different \% of remission
\end{tabular}

\section{Discussion}

In this study, we found male $66 \%$ and female $34 \%$, and most of the patients were in between 12-23 months. In a recent study, Britto et $\mathrm{al}^{16}$ showed that of the 123 children, 71 were males, 52 were females and 48 were in the age group 0-5 years. Developing countries have the largest number of disabled children below 15 years of age, estimated at $85 \%$ cases $^{16}$. In our study, we found girls with disabilities are less than the boys. The reason may be that parents are not coming with their girls because girls are less likely than boys to receive care and are more likely to be left out of family interactions and activities. Girls with disabilities are also less likely to get an education, receive vocational training, or find employment than are boys with disabilities or girls without disabilities ${ }^{17}$. In this study Tetraplegic $\mathrm{CP}$ with epilepsy was $58.0 \%$. A survey which was done in the year 2000 found $77.4 \%$ of the children identified with $\mathrm{CP}$ had spastic $\mathrm{CP}^{18}$. Approximately $60 \%$ of 8 -year-old children with $\mathrm{CP}$ had another developmental disability. More than $40 \%$ of children with CP had an intellectual disability, $35.0 \%$ had epilepsy, and more than $15.0 \%$ had vision impairment ${ }^{18}$.

Nearly 1 in 4 children with CP had both intellectual disability and epilepsy ${ }^{19}$. Our study differs from other studies where, almost all children with multiple disabilities had motor impairment (100.0\%). Developmental therapy and stimulation and neuro-rehabilitation approaches are important components in the treatment of children with CP. After intervention among 72 children, $92 \%$ improved in motor function. In a recent study Labaf et al showed significant improvement in rolling and laying, sitting, crawling, and standing abilities in children with spastic diplegia and quadriplegia after application of 
neurodevelopmental therapy ${ }^{20}$. Ketelaar et $\mathrm{al}^{21}$ showed significant differences in rolling, sitting, and kneeling after neurodevelopmental intervention. Fetters and Kluzik $^{22}$ reported that the use of neurodevelopmental treatment for children with $\mathrm{CP}$ improved motor skills functionality ${ }^{22}$.

Children develop rapidly during the first three years of life, so early detection and intervention are particularly important. Developmental screening is an effective means of detecting disability in children. It can take place in primary health-care settings, for example, during immunization visits or growth monitoring check-ups at community health centers. The purpose of screening is to identify children at risk, to refer them for further assessment and intervention as needed, and to provide family members with vital information on disability. Screening involves vision and hearing examinations as well as assessments of children's progress against such developmental milestones as sitting, standing, crawling, walking, talking, or handling objects. Parents can bring their children to Child Development Centers.

Child Development Centers (CDCs) have been established within government medical college tertiary hospitals across Bangladesh. Services entail a parent-professional partnership in a child and family-friendly environment with a focus on assessment, diagnosis, and management of a range of neurodevelopmental disorders in children and adolescents 0 to 16 years of age. Services are provided by a multidisciplinary team of professionals (child health physician, child psychologist, and developmental therapist) who emphasize the quality of services over the numbers of children seen.

\section{Conclusion}

This study shows that children with multiple disabilities are at risk of neurodevelopmental impairment. Regular follow up with appropriate therapy and intervention in different specialized clinic are effective in improvement of neurodevelopmental outcome of children with multiple disabilities.

\section{References}

1. Watson S. Teaching Students with Multiple Disabilities or Handicaps. Updated July 03, 2019

2. Horn E, Kang J. Supporting Young Children With Multiple Disabilities: What Do We Know and What Do We Still Need To Learn? Early Child Spec Educ. 2012; 31(4): 241-248

3. Scherzer AL, Tscharnuter I. Early Diagnosis and Therapy in Cerebral Palsy: A Primer on Infant Development Problems. 2nd
Ed. New York: Marcel Dekker Inc; 1990. 87-101

4. Hutton JL, Cooke T, Pharoah PO.Life expectancy in children with cerebral palsy. British Medical Journal 1994;13:430-435

5. Bulter C, Arrah J. Effects of neurodevelopmental treatment (NDT) for cerebral palsy: an AACPDM evidence report. Dev Med Child Neurol 2001;43(11):778-90

6. Ketelaar M, Vermeer A, Hart H, van Petegem-van Beek E, Helders PJM. Effects of a functional therapy program on motor abilities of children with cerebral palsy. PhysTher 2001;81:1534-1545

7. Mayston M. People with cerebral palsy: effects of and perspectives for therapy. Neural Plasticity 2001;8:51-69

8. Kerem G. Rehabilitation of children with cerebral palsy from a physiotherapist's perspective. Acta Orthop Traumatol Turc 2009;34(2):173-80

9. Shamsoddini AR, Hollisaz MT. effect of Sensory integration therapy on Gross motor Function in Children with cerebral palsy. Iran J Child Neurology 2009;8(1):43-48

10. Hathazi A. Interaction-based intervention programs in multiple disabilities. International Journal of Humanities and Social Science. 2014;4(12):135-9

11. Letchie $B$, Riggio $M$. Communication: Interactive Relationships in Alsop, L. Understanding Deafblindness. Issues, Perspectives, and Strategies, SKI-Hi Institute, Logan, Utah State University.2002;445- 466

12. Zeedyk MS, Caldwell P, Davies CE. How rapidly does Intensive Interaction promote social engagement for adults with profound learning disabilities? European Journal of Special Needs Education. 2009;24(2):119-37

13. Campbell SK. The child's development of functional movement. In: Campbell SK, Vander Linden DW, Palisano RJ, editors. Physical therapy for children. 2. Philadelphia: W. B. Saunders; 2000.3-44

14. Meaning and Definition of Multiple Disabilities. https://wecable.com/multiple-disabilities-meaning definition-example. Last updated: 29 May 2018, Published on: 29 May 2018 by the Team of Lalit Kumar

15. Khan NZ, Muslima H, Shilpi AB, Begum D, et al. Validation of rapid neurodevelopmental assessment for 2- to 5-year-old children in Bangladesh. Pediatrics 2013;131(2):486-94

16. Britto T, Poongothai A, Mamta A, Jesudasan N. Children with multiple disabilities and low vision: Identification, assessment, and training through low vision services in South India. International Congress Series 2005;1282: 42-46

17. The State of the World ${ }^{\text {ee }}$ Children. Children with Disabilities, 2013. Accessed on 1st May 2014

18. Ellen G, Adolescents, and Youth with Disabilities: Issues and challenges. Asia Pacific Disability Rehabilitation Journal July 2004; 15: 13-32

19. Bhasin TK, Brocksen S, Avchen RN, Van Naarden Braun K. Prevalence of four developmental disabilities among children aged 8 years - Metropolitan Atlanta Developmental Disabilities Surveillance Program. Surveillance Summaries 2000;55(1):1-9

20. Labaf N, Shamsoddini A, TaghiHollisaz M, Sobhani V, Shakibaee A. Effects of Neurodevelopmental Therapy on Gross Motor Function in Children with Cerebral Palsy. Iran J Child Neurol 2015; 9(2): 36-41

21. cts of a functional therapy program on motor abilities of children with cerebral palsy. Phys Ther 2001;81:1534-45

22. Fetters L, Kluzik J. The effects of neurodevelopmental treatment versus practice on the reaching of children with spastic cerebral palsy. Physical Therapy 1996;76(4):346-58 